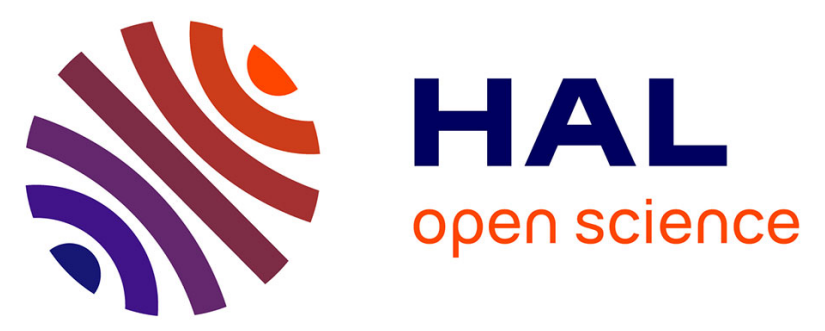

\title{
Selection of similar single domain antibodies from two immune VHH libraries obtained from two alpacas by using different selection methods.
}

\author{
Tengfei Li, Matthias Vandesquille, Sylvie Bay, Marc Dhenain, Benoît
}

Delatour, Pierre Lafaye

\section{To cite this version:}

Tengfei Li, Matthias Vandesquille, Sylvie Bay, Marc Dhenain, Benoît Delatour, et al.. Selection of similar single domain antibodies from two immune VHH libraries obtained from two alpacas by using different selection methods.. Immunology Letters, 2017, 188, pp.89-95. 10.1016/j.imlet.2017.07.001 . pasteur-01580563

\section{HAL Id: pasteur-01580563}

https://hal-pasteur.archives-ouvertes.fr/pasteur-01580563

Submitted on 1 Sep 2017

HAL is a multi-disciplinary open access archive for the deposit and dissemination of scientific research documents, whether they are published or not. The documents may come from teaching and research institutions in France or abroad, or from public or private research centers.
L'archive ouverte pluridisciplinaire HAL, est destinée au dépôt et à la diffusion de documents scientifiques de niveau recherche, publiés ou non, émanant des établissements d'enseignement et de recherche français ou étrangers, des laboratoires publics ou privés.

\section{(1)(1) $\$(0)$}

Distributed under a Creative Commons Attribution - NonCommercial - ShareAlikel 4.0 


\section{Accepted Manuscript}

Title: Selection of similar single domain antibodies from two immune VHH libraries obtained from two alpacas by using different selection methods

Authors: Tengfei Li, Matthias Vandesquille, Sylvie Bay, Marc Dhenain, Benoît Delatour, Pierre Lafaye

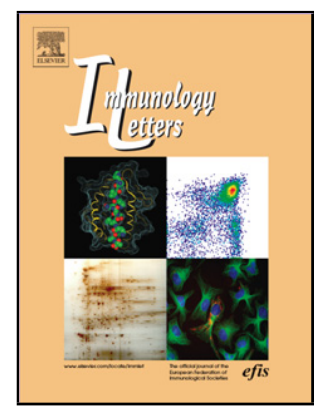

PII: S0165-2478(17)30116-5

DOI: http://dx.doi.org/doi:10.1016/j.imlet.2017.07.001

Reference: IMLET 6063

To appear in: Immunology Letters

Received date: 9-3-2017

Revised date: 14-6-2017

Accepted date: 2-7-2017

Please cite this article as: Li Tengfei, Vandesquille Matthias, Bay Sylvie, Dhenain Marc, Delatour Benoît, Lafaye Pierre.Selection of similar single domain antibodies from two immune VHH libraries obtained from two alpacas by using different selection methods.Immunology Letters http://dx.doi.org/10.1016/j.imlet.2017.07.001

This is a PDF file of an unedited manuscript that has been accepted for publication. As a service to our customers we are providing this early version of the manuscript. The manuscript will undergo copyediting, typesetting, and review of the resulting proof before it is published in its final form. Please note that during the production process errors may be discovered which could affect the content, and all legal disclaimers that apply to the journal pertain. 


\section{Selection of similar single domain antibodies from two immune VHH libraries obtained from two alpacas by using different selection methods}

Tengfei Li ${ }^{\mathrm{a}, \mathrm{b}}$, c, Matthias Vandesquille ${ }^{\mathrm{d}}$, Sylvie Bay ${ }^{\mathrm{d}, \mathrm{e}}$, Marc Dhenain ${ }^{\mathrm{f}}$, Benoît Delatour ${ }^{\mathrm{b}}$ and Pierre Lafaye ${ }^{\mathrm{a}, *}$

${ }^{a}$ Institut Pasteur, CITECH, Plateforme d'Ingénierie des Anticorps, Paris, France

b Sorbonne Universités, UPMC Univ. Paris 06 UMR S 1127, Inserm U 1127/ CNRS UMR 7225, Paris, France

${ }^{c}$ Université Paris Diderot-Paris 7, Paris, France

d Institut Pasteur, Unité de Chimie des Biomolécules, Paris, France

e CNRS UMR 3523, Paris, France

${ }^{\mathrm{f}}$ Commissariat à l'Energie Atomique et aux Energies Alternatives (CEA), Direction de la Recherche Fondamentale (DRF), MIRCen, CNRS, Université Paris-Sud, Université Paris-Saclay UMR 9199, Neurodegenerative Diseases Laboratory, Fontenay-aux-Roses, France

E-mail address : plafaye@pasteur.fr

\section{HIGHLIGHTS}

- VHHs against GFAP have been obtained by Ribosome display after immunization of one alpaca with GFAP

- VHHs against GFAP have been obtained by phage display after immunization of another alpaca with a human brain extract containing GFAP

- All the VHHs were closely related and one VHH was found to be strictly identical in both studies.

\section{ABSTRACT}

The two most used methods to select camelid single-domain antibody-fragments 
(VHHs) are: displaying their repertoires on the surface of filamentous bacteriophages (phage display) or linking them to ribosomes (ribosome display). In this study, we compared specific VHHs isolated from two different immune libraries coming from two different alpacas by using these two selection methods.

Three anti-GFAP (glial fibrillary acidic protein) VHHs were derived from an immune library obtained by ribosome display after immunization of one alpaca with purified GFAP, a protein expressed by astroglial cells. In parallel, three other antiGFAP VHHs were derived from an immune library by phage display after immunization of another alpaca with a human brain tissue extract containing GFAP. All the VHHs were closely related and one VHH was found to be strictly identical in both studies. This highlights the selection pressure exerted by the camelid immune system to shape the paratope of an antibody against a defined antigen.

Keywords.: Anti GFAP VHH, VH, VHH, phage display, ribosome display.

\section{Introduction}

Besides producing conventional tetrameric antibodies, camelids also display functional antibodies composed of homodimerized heavy chains. These antibodies lacking light chains, interact with antigens by the virtue of only one single variable domain of the heavy chain, referred to as VHH to distinguish it from $\mathrm{VH}$ of conventional antibodies [1]. VHHs possess a number of advantages for antibody engineering when compared with the conventional antibody fragments (Fab: Fragment antigen-binding or scFv: Single-chain variable fragment). VHHs are highly soluble and can be expressed at high levels in the periplasm and the cytoplasm of $E$. coli and eukaryotic cells [2,3]. Due to their relatively small size (14-17 kDa), VHHs offer also many advantages in terms of structural analysis and in diagnostic applications requiring tissue penetration [2-9]. Achour I et al, 2008 [10] demonstrated 
that a single immunoglobulin heavy chain locus in alpaca chromosome 4 contains all of the genetic elements required for generation of the two types of antibodies. Phage and ribosome display are ideal tools of widespread use for the selection of different antibody fragments [11,12]. Phage display was first described by Smith [13] and McCafferty et al [14] used it successfully for the selection of antibodies for the first time in 1990. Compared with other display technologies, phage display possesses many advantages: robustness, simplicity and stability of the phage particles and the selection can be performed on cell surface, tissue sections and in vivo [15-18]. However, phage-display libraries sizes are restricted to $10^{10}-10^{11}$ by the efficiency of bacterial transformation. In vitro display technologies have since been developed as an alternative, the most widely used being ribosome display [19]. Hanes et al successfully selected antibody fragments by using this method in 1998 and 2000 [20,21]. Despite many improvements made recently, ribosome display is still challenging to perform for selection on cell surfaces or against non-purified antigens. On the other side, a strong advantage of ribosome display over phage display, is that the encoded DNA does not need to be imported into a host because DNA amplification and protein synthesis occurs totally in vitro. As a consequence, ribosome display libraries can allow more diversity with possibly more than $10^{13}$ binders [12].

Here we wanted to compare VHHs directed against the same protein obtained from two immune libraries by using the two pre-cited most-used selection techniques for antibody screening. The antigen of interest was GFAP (glial fibrillary acidic protein), a well-known marker of astrocytes. Two alpacas were immunized either with a human brain extract that contained GFAP as well as numerous other proteins/peptides, or with purified human GFAP. Then immune libraries were prepared from each animal. Using phage display or ribosome display applied to the two respective different immune libraries, we have selected closely related anti-GFAP VHHs. Unexpectedly one common VHH was isolated from these two different libraries. This emphasizes the strength of selection pressure exerted by the camelid immune system to shape the paratope of an antibody against a defined and highly specific antigen and highlights 
that selection of a relevant VHH against an epitope is not always dependent on the selection method.

\section{Materials and methods}

\subsection{Immunization}

Postmortem brain tissue (hippocampal region) from a patient with Alzheimer's disease was obtained from the NeuroCEB brain bank. This bank is associated to a brain donation program run by a consortium of patient associations and declared to the Ministry of Research and Universities, as requested by French Law. An explicit written consent was obtained for the brain donation in accordance with the French Bioethical Laws. A brain homogenate was performed with this brain sample according to Mercken M et al 1992 [22]. Briefly, tissues are homogenized in $10 \mathrm{mM}$ Tris, $1 \mathrm{mM}$ EGTA, $0.8 \% \mathrm{NaCl}, 10 \%$ sucrose buffer containing protease and phosphatase inhibitors (Pierce), followed by centrifugation at $27,000 \mathrm{~g}$ for $20 \mathrm{~min}$. $1 \%$ Beta-mercaptoethanol and 1\% N-Laurylsarcosine is added to the supernatant and the mixture is incubated for $2.5 \mathrm{~h}$ at $37^{\circ} \mathrm{C}$. After centrifugation at $100,000 \mathrm{~g}$ for $35 \mathrm{~min}$, the pellet is homogeneized in PBS (Phosphate-buffered saline) at a concentration of 1 $\mathrm{mg} / \mathrm{ml}$. This human brain extract is referred to as $\mathrm{Sg} 4697$. One alpaca was injected with $90 \mu \mathrm{l}$ of the mixture mixed with $90 \mu \mathrm{l}$ of Freund complete adjuvant for the first immunization, and with $90 \mu \mathrm{l}$ of Freund incomplete adjuvant for the following immunizations. Another alpaca was previously immunized with purified human GFAP (USBiological) [8]. In France, an ethical approval is not required for immunization of camelids with Freund (in-) complete adjuvant. The immune responses were monitored by titration of serum samples by ELISA on coated GFAP. The bound alpaca antibodies were detected with polyclonal rabbit anti-alpaca IgGs [23]. The same procedure was performed on a murine brain extract. The extract was referred to as mWT45.

\subsection{Library construction, selection and expression of $\mathrm{VHH}$} The blood of the animal immunized with the human brain extract was collected. The selection of cDNA coding only for $\mathrm{VHH}$ were realized according to Lafaye et al, 
2009, 1995 [23,24]. The $v h h$ genes were then cloned into phagemid vector pHEN1 by using primers contained enzymatic NcoI and NotI restriction sites at the 5' and 3'ends, respectively. The size of the library was $3 \times 10^{8} \mathrm{cfu}$.

Phage display protocols were performed as described in Lafaye et al, 2009 [23]. Briefly $10^{13}$ phage-VHH diluted in PBS were used to perform a panning cycle by using saturated Nunc immunotubes (InterMed) coated with human brain extract (10 $\mu \mathrm{g} / \mathrm{ml})$. To increase the stringency, different blocking buffers for each panning were used: $2 \%$ skimmed milk as saturating agent for the first round; 5\% BSA (Bovine Serum Albumin) and Odyssey blocking buffer (LI-COR Biosciences) diluted at $1 / 4$ for the second and third one.

Sixty-one individual clones were randomly chosen and tested by ELISA against Sg4697 and BSA. The DNA corresponding to the positive VHHs was sequenced by GATC Biotech. The sequences were processed with DNA strider and analyzed using ClustalW2-Multiple Sequence Alignment of EMBL-EBI.

The blood of the animal immunized with GFAP was collected and selection of cDNA coding for $\mathrm{VHH}$ was performed as described earlier. The $v h h$ population was converted to ribosome display format using PCR and transcribed to mRNA [8]. Selection of ternary complexes involving mRNA, ribosome and functional VHH was performed on GFAP as described by Perruchini et al, 2009 [8].

The VHH sequences were analyzed at the genomic level using IMGT (International ImMunoGeneTics information system) alpaca genomic database.

To summarize, the VHHs A12, G110, C5F7, C7G7, B70, B7, G11 and H8 were obtained by phage display, the VHHs E9, E3 and A10 were obtained by ribosome display.

Specific $v h h$ genes were subcloned in a modified pASK-IBA2 vector (Genosys Iba) containing the NcoI and NotI restriction sites. pASK-IBA2 allows the periplasmic expression of a strep-tag at the C-terminal end in frame with the VHH. Transformed $E$. coli XL2-Blue ultracompetent cells expressed VHHs in the periplasm after induction by anhydrotetracycline $(200 \mu \mathrm{g} / \mathrm{l})$ for $16 \mathrm{~h}$ at $20^{\circ} \mathrm{C}$. After centrifugation, cells were resuspended in $100 \mathrm{mM}$ Tris- $\mathrm{HCl} \mathrm{pH} 8,150 \mathrm{mM} \mathrm{NaCl}, 1 \mathrm{mM}$ EDTA, then lysed by 
polymyxin B sulfate $\left(1 \mathrm{mg} / \mathrm{ml}\right.$ Fluka) for $1 \mathrm{~h}$ at $4^{\circ} \mathrm{C}$, the periplasmic extract being obtained after centrifugation. Purified VHHs were obtained by Strep-Tactin Sepharose column (Iba) according to the manufacturer's instructions.

\subsection{ELISA}

Microtitre plates (Nunc) were incubated with $1 \mu \mathrm{g} / \mathrm{mL}$ of GFAP diluted in PBS overnight at $4^{\circ} \mathrm{C}$. Plates were washed 4 times with PBS containing $0.1 \%(w / v)$ Tween-20 (buffer A) and saturated with buffer A containing 0,5\% (w/v) gelatin (buffer B) for $30 \mathrm{~min}$. Sera were then added. Plates were incubated for $1 \mathrm{~h}$ at $37^{\circ} \mathrm{C}$ and washed prior the addition of rabbit anti-alpaca Ig followed by peroxidase conjugated goat anti-rabbit Igs (ICN biomedicals).

A competition assay was performed to determine VHH-binding sites. Various concentrations of His-Tagged VHH H8/E9 was incubated into wells coated with GFAP for $1 \mathrm{~h}$ at $37^{\circ} \mathrm{C}$. Plates were washed and strep-tagged VHHs (H8/E9, B7 and G11) were added. After additional incubation for $1 \mathrm{~h}$ at $37^{\circ} \mathrm{C}$, the presence of the strep-tagged VHHs was revealed by the addition of an home-made anti-strep tag mAb (eBioscience) followed by peroxidase-conjugated goat anti mAb (ICN biomedicals).

\subsection{Western Blot}

To control the VHH specificity by WB, $10 \mu 1$ of diluted (1:10) Sg4697 human and mWT45 mouse brain extracts and $1 \mu \mathrm{g}$ of human GFAP (USBiological) were used. Protein samples were treated at $70^{\circ} \mathrm{C}$ for $10 \mathrm{~min}$ in reducing conditions. Following the separation by polyacrylamide gel electrophoresis (PAGE) using NuPAGE Novex 4$12 \%$ Bis-Tris gels, semi-dry transfer onto Hybond-C (Amersham) was performed, the membranes were blocked in a $4 \%$ skimmed milk solution. Purified strep-tagged VHHs $(1 \mu \mathrm{g} / \mathrm{ml})$ were used as primary antibodies at $4^{\circ} \mathrm{C}$ overnight. An in-house antistrep tag mouse mAb (C23-21) was added at $1 \mu \mathrm{g} / \mathrm{ml}$ and incubated for $1 \mathrm{~h}$ at $37^{\circ} \mathrm{C}$. Peroxydase-labeled goat anti-Mouse Ig 1:10000 (Vector) was added and incubated for $1 \mathrm{~h}$ at $37^{\circ} \mathrm{C}$. Finally, peroxidase activity was visualized using a chemiluminescent kit (Amersham).

\subsection{Immunohistochemistry}

Adult C57BL6 mice were euthanized by intraperitoneal injection of sodium 
pentobarbital (CEVA). Brains were then fixed by intracardiac perfusion of $4 \%$ paraformaldehyde in PBS, and postfixed in the same fixative overnight at $4{ }^{\circ} \mathrm{C}$. Forty micrometer-thick coronal brain sections were then prepared in PBS using a vibratome (Leica VT1000S). The sections were pretreated for 10 min with $1 \%$ hydrogen peroxide to neutralize endogenous peroxidase. Non-specific binding was blocked by incubation of the sections for 40 min with Odyssey blocking buffer (LI-COR Biosciences) supplemented with $0.2 \%$ Triton X-100. The sections were then incubated with $1 \mu \mathrm{g} / \mathrm{ml} \mathrm{VHH-Strep-tag} \mathrm{overnight} \mathrm{at} 4^{\circ} \mathrm{C}$ in the same blocking buffer. After washing in PBS $0.2 \%$ Triton, sections were then incubated with biotinylated anti-strep tag mAb C23-21 at $1 \mu \mathrm{g} / \mathrm{ml}$ for $1 \mathrm{~h}$, followed by ABC complex (Vector) for $30 \mathrm{~min}$, and then with DAB (Diaminobenzidine) at $0.5 \mathrm{mg} / \mathrm{ml}$ as chromogen. After DAB processing, sections were collected on superfrost glass slides, air dried at room temperature overnight, and then dehydrated by placing the slides in graded ethanol solutions (5 min in $70 \%$ and $90 \%$ ethanol respectively and 5 min in 100\% ethanol twice) and xylene (5 min 3 times), and finally mounted in Eukitt mounting medium (O. Kindler GmbH).

\subsection{Measurement of VHH affinity}

Affinity was measured in solution $[8,25]$. Briefly, VHH samples were incubated overnight with increasing amounts of GFAP (Millipore) at concentrations ranging from $10^{-11}$ to $10^{-7} \mathrm{M}$. The remaining free antigen binding sites were then quantified by ELISA using an anti-His tag $\mathrm{mAb}$, followed by an anti-mouse $\beta$-galactosidase conjugate and 4-methylumbelliferyl $\beta$-D galactoside substrate. Fluorescence was read at $460 \mathrm{~nm}$ after excitation at $355 \mathrm{~nm}$. KD was estimated from the slope of the regression curve obtained by plotting the reciprocal of the fraction bound antibody versus the reciprocal of the molar concentration of the antigen. 


\section{Results}

3.1. Obtention of VHHs using phage or ribosome display and comparison of their sequences

One alpaca was immunized with human brain extract from an Alzheimer's patient presenting severe brain neuroinflammation, including reactive astrogliosis. A VHH phage display library was prepared and the selection of clones was performed against the same human brain extract. After 3 rounds of panning, 221 clones were tested in ELISA against the brain extract and BSA (a non-relevant antigen) to discriminate specific from non-specific clones. Sixty-one clones were identified as positive, 34 were sequenced, revealing only 8 different sequences, A12, G110, C5F7, C7G7, B70, B7, G11 and H8 (Fig. 1).

A12 appeared most frequently with 19 occurrences and G110 was found 5 times. C5F7 and C7G7 appeared both 3 times. B70 was detected only once. B7, G11and H8 (one occurrence each) share the same CDRs.

We analyzed the biochemical properties of these VHHs obtained from the phage display library. After cloning in pASK vector and periplasmic expression, the purified VHHs with a strep-tag at the $C$ - terminus were obtained. Until now, for A12, B70, C7G7, G110 and C5F7 no accurate antigen target has been identified in immunohistochemistry using mouse brain tissue.

Interestingly, we found that the new VHHs B7, H8 and G11 obtained by phage display are closely related to the anti-GFAP VHHs previously isolated after ribosome display screening. Indeed, another alpaca was immunized with purified human GFAP. Lymphocytes and VHH corresponding DNA were isolated, the VHH-coding mRNA sequences were selectively amplified. Three VHHs (E9, E3 and A10) with a high affinity for GFAP and their corresponding cDNA were selected by ribosome display [8] (Fig. 2). Also, one VHH with strictly identical sequence was isolated by the two procedures (H8 and E9 [8], respectively) (Fig. 1).

As the recovery of a same VHH from two different animals with two different methods was totally unexpected we then analyzed the polyclonal serum of both alpacas for their ability to recognize GFAP by ELISA (Fig. 3). Notably, the serum of 
the alpaca immunized with the brain extract did recognize GFAP, although with a slight reactivity, while the polyclonal serum of the alpaca immunized with GFAP presented a high titer of anti-GFAP, as expected. In parallel we used a polyclonal serum taken from a third alpaca which had never been immunized with human GFAP. No GFAP recognition was observed with this serum. The low titer of GFAP-specific antibodies in the serum of the animal immunized with human brain extract also emphasized the efficiency of the phage display selection method to recover minor clones.

\subsection{Biochemical characterization of the anti GFAP VHHs}

Western blot experiment was performed with VHHs H8/E9, B7 and G11. Three samples were analysed: human brain extract (Sg4697), C57B/6 adult wild type mouse brain extract (mWT45) and the purified GFAP. WB analysis showed that the different VHHs labelled several bands between 35 and $55 \mathrm{kDa}$ in human brain extract (Fig. 4A). Similar outcomes had already been observed by immunoblot using anti-GFAP antibodies on human brain extract, due to different isoforms of the protein in humans $[8,26]$. The VHHs labelled one band just below $55 \mathrm{kDa}$ in mouse brain extract, in accordance with the fact that only one main isoform is expressed in mice (Fig. 4A). They also recognized the GFAP protein as a positive control (Fig. 4B). To determine whether the VHHs recognized the same epitope, competition among the 3 VHHs for binding to GFAP was carried out as described in "Materials and Methods". The binding of strep-tagged VHHs decreased as a function of increasing concentrations of the his-tagged VHH H8/E9 (Fig 5), suggesting that VHHs recognize the same epitope, or at least closely related epitopes, on GFAP.

H8/E9, B7 and G11 obtained either after immunization with purified human GFAP or with human brain extract strongly and specifically immunodetected GFAP in astrocytes in mouse brain tissue (Fig. 6), which was normally expected, as mouse GFAP protein showed $91 \%$ sequence identity to that of human [27].

The affinity of VHHs obtained by the two methods was measured by ELISA $[8,25]$. A10, E9, H8 and G11 had affinities in the nanomolar range while the affinity of E3 
was in the micromolar range and $\mathrm{B} 7$ had an affinity of $7.1 \times 10^{-10} \mathrm{M}, 8$-fold higher than the affinity of VHH H8 and VHH E9 respectively (Table 1).

For E3, the presence of a proline at position 25 instead of a serine probably induced a modification of the CDR1 loop as proline is usually found in sharp turns [8,28]. This modification could alter E3 functionality as reflected by the sharp decrease of its affinity for GFAP. B7 had a higher affinity when compared to other VHHs. The differences between B7 and both VHHs H8 and E9 were Leu11, Glu13 and Ala89 in B7 instead of Ser11, Gln13 and Glu89 in H8/E9 (Fig. 1), This was associated to local charge modifications in B7: an extra negative charge introduced at position 13 and a negative charge removed at position 89 . These features might positively influence VHH binding by modifying the structure of CDRs or by involving some amino acids of framework (FR) in interacting with the antigen.

\subsection{Obtaining VHHs with VH characteristics}

The gene segment families were identified by a search for similarities in IMGT. The nucleotide sequence of VHHs showed a $90 \%$ sequence similarity with a single V gene referred to as IGHV3S31 (accession number AM939730 [10]) which is classified as a VH gene [10] The junction segment JH of VHH corresponded to IGHJ4 genetic element [29]. No D gene was found according to IMGT analysis.

Obtaining VH from immune VHH libraries was unexpected, but some studies demonstrated that a minority (5-10\%) of VHH originates from VH germline genes, which have been identified together with canonical VHH in immune libraries [7,30]. In FR2 regions, VH possess the conserved Val37, Gly44, Leu45 and Trp47, which are clustered in space and these $\mathrm{VH}$ amino acid hallmarks form a hydrophobic bond with the VL [31,32]. In VHH, these amino acids are often substituted (Val37Phe or Val37Tyr, Gly44Glu, Leu45Arg and Trp47Gly) [33]. We found for all anti-GFAP VHHs Gly44 and Trp47 which are VH conserved amino acids. At positions 37 and 45, instead of a valine and a leucine we respectively found a leucine and an isoleucine which are closely related amino-acids (Fig. 1). Moreover Trp118 was substituted by an arginine, a feature usually observed when conventional VH3 genes recombine with a D-JH and are expressed as homodimeric IgG $[34,35]$. 


\section{Discussion}

Similar VHHs directed against GFAP were isolated with two different selection methods and from two different animals immunized with two different antigenic preparations based on GFAP-containing human brain extract $v s$ purified GFAP (Fig. 2)

We can exclude possible contamination between the two immune libraries, first because experiments were not performed simultaneously. The selection of $\mathrm{H} 8$ by phage display is recent while the selection of VHH E9 by ribosome display with the other immune library was made previously [8]. Also the selection of the two antibodies was performed using a completely distinct process with no common reagents or common steps.

Using an immune library is an important factor for successful selection and retrieval of high affinity antibodies [15]. Following the immunization with an antigen, the specific B cells will proliferate and undergo affinity maturation for the antigen. Mature immunoglobulins (Igs) result from a maturation process in which multiple recombinations and mutations occur. It is estimated that in humans the repertoire of Igs expressed by naive B cells could contain as many as $10^{11}$ different Igs. Moreover, somatic hypermutation brings in point mutations into the rearranged $\mathrm{V}$-region genes of activated B cells, generating further diversity that can be selected by its enhanced antigen binding properties [36]. As camelids share similar VDJ recombination process with humans during B cell ontogeny[10], we could expect comparable diversity for homodimeric camelid IgGs. Among all variants generated during this maturation, only the best ones are selected. Despite the wide variety possibilities it is surprising that closely related VHHs could be selected. Pellis M et al, 2012 [15] succeeded to select similar HIV-I integrase -specific VHHs by using concurrently bacterial-twohybrid selection system (BH VHHs) and conventional phage display panning (PD VHHs) but selections were performed using a unique immune library. The comparison between 13 BH VHHs sequences and 12 PD VHHs sequences reveals 
that 5 of BH VHHs are extremely similar to PD VHHs, some VHHs obtained from these two different selection methods have the same CDR3. Even more, one BH VHH and one PD VHH share an exactly identical sequence.

In our case it is astonishing that all six VH/VHHs share almost the same FRs and CDRs with only a few amino-acid modifications, that demonstrates only one $v h / v h h$ gene was selected and the affinity maturation of these VH/VHHs go through almost an identical process. So the recovery of closely related VHHs from 2 different libraries derived from 2 animals with 2 selection techniques, at least in our case could not be just a coincidence. It emphasizes the existence of an immunodominant epitope within GFAP, which could be recognized by only a very limited number of VH/VHH possibilities.

\section{Acknowledgments}

We thank the GIE Neuro-CEB of the hospital Pitié-Salpêtrière for providing human brain tissue. We would like to thank Yohann Dickx for his technical help. We thank Patrick England for the final proofreading of the manuscript. We gratefully acknowledge sequence analysis from IMGT (the International ImMunoGeneTics information), Montpellier, France. The immunohistochemistry of this work was carried out on the HISTOMICS platform of the ICM and we sincerely acknowledge all staff involved.

\section{Potential conflict of interest}

No potential conflicts of interest were disclosed.

\section{Authorship}

TL and PL conceived and designed the research, analyzed data, interpreted results of experiments, prepared the figures, drafted the manuscript and approved the final version of the manuscript; TL performed experiments; TL, MV, SB, MD, BD and PL edited and revised manuscript. 


\section{References}

[1] C. Hamers-Casterman, T. Atarhouch, S. Muyldermans, G. Robinson, C. Hamers, E.B. Songa, N. Bendahman, R. Hamers, Naturally occurring antibodies devoid of light chains., Nature. 363 (1993) 446-8. doi:10.1038/363446a0.

[2] T. Li, J.-P. Bourgeois, S. Celli, F. Glacial, A.-M. Le Sourd, S. Mecheri, B. Weksler, I. Romero, P.-O. Couraud, F. Rougeon, P. Lafaye, Cell-penetrating anti-GFAP VHH and corresponding fluorescent fusion protein VHH-GFP spontaneously cross the blood-brain barrier and specifically recognize astrocytes: application to brain imaging., FASEB J. 26 (2012) 396979. doi:10.1096/fj.11-201384.

[3] S. Muyldermans, Nanobodies: natural single-domain antibodies., Annu. Rev. Biochem. 82 (2013) 775-97. doi:10.1146/annurev-biochem-063011-092449.

[4] A. Olichon, T. Surrey, Selection of genetically encoded fluorescent single domain antibodies engineered for efficient expression in Escherichia coli., J. Biol. Chem. 282 (2007) 36314-20. doi:10.1074/jbc.M704908200.

[5] M. Arbabi Ghahroudi, A. Desmyter, L. Wyns, R. Hamers, S. Muyldermans, Selection and identification of single domain antibody fragments from camel heavy-chain antibodies., FEBS Lett. 414 (1997) 521-6. doi:10.1016/S0014-5793(97)01062-4.

[6] S. a Jobling, C. Jarman, M.-M. Teh, N. Holmberg, C. Blake, M.E. Verhoeyen, Immunomodulation of enzyme function in plants by single-domain antibody fragments., Nat. Biotechnol. 21 (2003) 77-80. doi:10.1038/nbt772.

[7] J. Tanha, G. Dubuc, T. Hirama, S. a Narang, C.R. MacKenzie, Selection by phage display of llama conventional $\mathrm{V}(\mathrm{H})$ fragments with heavy chain antibody $\mathrm{V}(\mathrm{H}) \mathrm{H}$ properties., J. Immunol. Methods. 263 (2002) 97-109. doi.org/10.1016/S0022-1759(02)00027-3.

[8] C. Perruchini, F. Pecorari, J.-P. Bourgeois, C. Duyckaerts, F. Rougeon, P. Lafaye, Llama VHH antibody fragments against GFAP: better diffusion in fixed tissues than classical monoclonal antibodies., Acta Neuropathol. 118 (2009) 685-95. doi:10.1007/s00401-0090572-6.

[9] T. Li, M. Vandesquille, F. Koukouli, C. Dudeffant, I. Youssef, P. Lenormand, C. Ganneau, U. Maskos, C. Czech, F. Grueninger, C. Duyckaerts, M. Dhenain, S. Bay, B. Delatour, P. Lafaye, 
Camelid single-domain antibodies: A versatile tool for in vivo imaging of extracellular and intracellular brain targets., J. Control. Release. 243 (2016) 1-10. doi:10.1016/j.jconrel.2016.09.019.

[10] I. Achour, P. Cavelier, M. Tichit, C. Bouchier, P. Lafaye, F. Rougeon, Tetrameric and homodimeric camelid IgGs originate from the same IgH locus., J. Immunol. 181 (2008) 2001-9. doi.org/10.4049/jimmunol.181.3.2001.

[11] J.-L. Jestin, P. Lafaye, Parallel Processing of Complex Biomolecular Information: Combining Experimental and Computational Approaches, in: Syst. Comput. Biol. Bioinforma. Comput. Model., InTech, 2011: pp. 1-22. doi:10.5772/22506.

[12] P. Dufner, L. Jermutus, R.R. Minter, Harnessing phage and ribosome display for antibody optimisation., Trends Biotechnol. 24 (2006) 523-9. doi:10.1016/j.tibtech.2006.09.004.

[13] G.P. Smith, Filamentous fusion phage: novel expression vectors that display cloned antigens on the virion surface., Science. 228 (1985) 1315-7. doi: 10.1126/science.4001944.

[14] J. McCafferty, A.D. Griffiths, G. Winter, D.J. Chiswell, Phage antibodies: filamentous phage displaying antibody variable domains., Nature. 348 (1990) 552-4. doi:10.1038/348552a0.

[15] M. Pellis, E. Pardon, K. Zolghadr, U. Rothbauer, C. Vincke, J. Kinne, I. Dierynck, K. Hertogs, H. Leonhardt, J. Messens, S. Muyldermans, K. Conrath, A bacterial-two-hybrid selection system for one-step isolation of intracellularly functional Nanobodies., Arch. Biochem. Biophys. 526 (2012) 114-23. doi:10.1016/j.abb.2012.04.023.

[16] B. Liu, F. Conrad, M.R. Cooperberg, D.B. Kirpotin, J.D. Marks, Mapping tumor epitope space by direct selection of single-chain Fv antibody libraries on prostate cancer cells., Cancer Res. 64 (2004) 704-10. doi:10.1158/0008-5472.CAN-03-2732.

[17] J. Tordsson, L. Abrahmsén, T. Kalland, C. Ljung, C. Ingvar, T. Brodin, Efficient selection of scFv antibody phage by adsorption to in situ expressed antigens in tissue sections., J. Immunol. Methods. 210 (1997) 11-23. doi.org/10.1016/S0022-1759(97)00165-8.

[18] R. Pasqualini, E. Ruoslahti, Organ targeting in vivo using phage display peptide libraries., Nature. 380 (1996) 364-6. doi:10.1038/380364a0.

[19] J. Hanes, A. Plückthun, In vitro selection and evolution of functional proteins by using ribosome display., Proc. Natl. Acad. Sci. U. S. A. 94 (1997) 4937-42. 
https://www.ncbi.nlm.nih.gov/pubmed/9144168.

[20] J. Hanes, L. Jermutus, S. Weber-Bornhauser, H.R. Bosshard, A. Plückthun, Ribosome display efficiently selects and evolves high-affinity antibodies in vitro from immune libraries., Proc. Natl. Acad. Sci. U. S. A. 95 (1998) 14130-5. doi:10.1073/pnas.95.24.14130.

[21] J. Hanes, C. Schaffitzel, A. Knappik, A. Plückthun, Picomolar affinity antibodies from a fully synthetic naive library selected and evolved by ribosome display., Nat. Biotechnol. 18 (2000) 1287-92. doi:10.1038/82407.

[22] M. Mercken, M. Vandermeeren, U. Lübke, J. Six, J. Boons, A. Van de Voorde, J.J. Martin, J. Gheuens, Monoclonal antibodies with selective specificity for Alzheimer Tau are directed against phosphatase-sensitive epitopes., Acta Neuropathol. 84 (1992) 265-72. http://www.ncbi.nlm.nih.gov/pubmed/1384266.

[23] P. Lafaye, I. Achour, P. England, C. Duyckaerts, F. Rougeon, Single-domain antibodies recognize selectively small oligomeric forms of amyloid beta, prevent Abeta-induced neurotoxicity and inhibit fibril formation., Mol. Immunol. 46 (2009) 695-704. doi:10.1016/j.molimm.2008.09.008.

[24] P. Lafaye, F. Nato, J.C. Mazié, N. Doyen, Similar binding properties for a neutralizing antitetanus toxoid human monoclonal antibody and its bacterially expressed Fab., Res. Immunol. 146 (1996) 373-82. http://www.ncbi.nlm.nih.gov/pubmed/8739329.

[25] B. Friguet, A.F. Chaffotte, L. Djavadi-Ohaniance, M.E. Goldberg, Measurements of the true affinity constant in solution of antigen-antibody complexes by enzyme-linked immunosorbent assay., J. Immunol. Methods. 77 (1985) 305-19. doi:10.1016/00221759(85)90044-4.

[26] M.A. Korolainen, S. Auriola, T.A. Nyman, I. Alafuzoff, T. Pirttilä, Proteomic analysis of glial fibrillary acidic protein in Alzheimer's disease and aging brain., Neurobiol. Dis. 20 (2005) 858-70. doi:10.1016/j.nbd.2005.05.021.

[27] K. Eun, S.-U. Hwang, H.-M. Jeon, S.-H. Hyun, H. Kim, Comparative Analysis of Human, Mouse, and Pig Glial Fibrillary Acidic Protein Gene Structures., Anim. Biotechnol. 27 (2016) 126-32. doi:10.1080/10495398.2015.1126719.

[28] a Desmyter, T.R. Transue, M. a Ghahroudi, M.H. Thi, F. Poortmans, R. Hamers, S. Muyldermans, L. Wyns, Crystal structure of a camel single-domain VH antibody fragment 
in complex with lysozyme., Nat. Struct. Biol. 3 (1996) 803-11.

http://www.ncbi.nlm.nih.gov/pubmed/8784355.

[29] V.K. Nguyen, S. Muyldermans, R. Hamers, The specific variable domain of camel heavychain antibodies is encoded in the germline., J. Mol. Biol. 275 (1998) 413-8. doi:10.1006/jmbi.1997.1477.

[30] A. Monegal, A. Olichon, N. Bery, T. Filleron, G. Favre, A. de Marco, Single domain antibodies with VH hallmarks are positively selected during panning of llama (Lama glama) naïve libraries., Dev. Comp. Immunol. 36 (2012) 150-6. doi:10.1016/j.dci.2011.06.016.

[31] S. Muyldermans, C. Cambillau, L. Wyns, Recognition of antigens by single-domain antibody fragments: the superfluous luxury of paired domains., Trends Biochem. Sci. 26 (2001) 230-5. dx.doi.org/10.1016/S0968-0004(01)01790-X.

[32] K.B. Vu, M.A. Ghahroudi, L. Wyns, S. Muyldermans, Comparison of llama VH sequences from conventional and heavy chain antibodies., Mol. Immunol. 34 (1997) 1121-31. doi:10.1016/S0161-5890(97)00146-6.

[33] S. Muyldermans, T. Atarhouch, J. Saldanha, J. a Barbosa, R. Hamers, Sequence and structure of VH domain from naturally occurring camel heavy chain immunoglobulins lacking light chains., Protein Eng. 7 (1994) 1129-35. http://www.ncbi.nlm.nih.gov/pubmed/7831284.

[34] M.M. Harmsen, R.C. Ruuls, I.J. Nijman, T. a Niewold, L.G. Frenken, B. de Geus, Llama heavychain $\mathrm{V}$ regions consist of at least four distinct subfamilies revealing novel sequence features., Mol. Immunol. 37 (2000) 579-90. doi.org/10.1016/S0161-5890(00)00081-X.

[35] D. Saerens, J. Kinne, E. Bosmans, U. Wernery, S. Muyldermans, K. Conrath, Single domain antibodies derived from dromedary lymph node and peripheral blood lymphocytes sensing conformational variants of prostate-specific antigen., J. Biol. Chem. 279 (2004) 51965-72. doi:10.1074/jbc.M409292200.

[36] C.A.J. Janeway, P. Travers, M. Walport, The generation of diversity in immunoglobulins Virtually, Garland Science, New York, 2001. https://www.ncbi.nlm.nih.gov/books/NBK27140. 
Figure legends

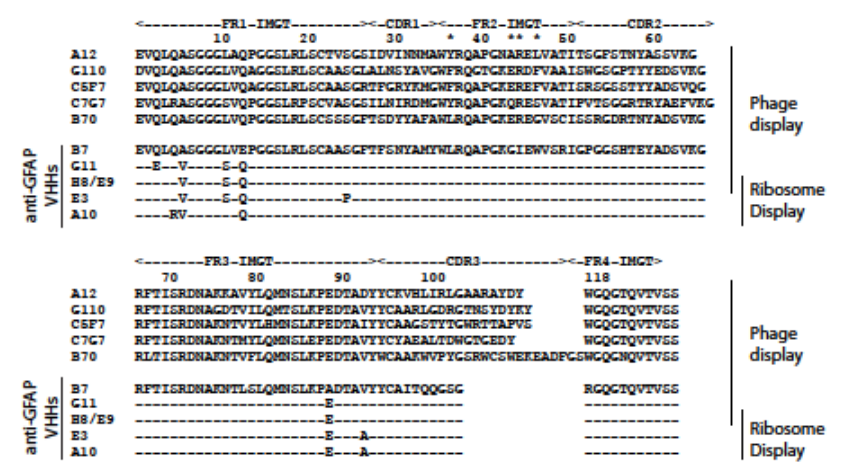

Fig. 1. Sequence of anti-GFAP VHHs obtained by concurrent phage/ribosome display methods. Dotted lines indicate identical sequences. CDRs were determined according to IMGT. * indicated VH hallmarks present in FR2. 

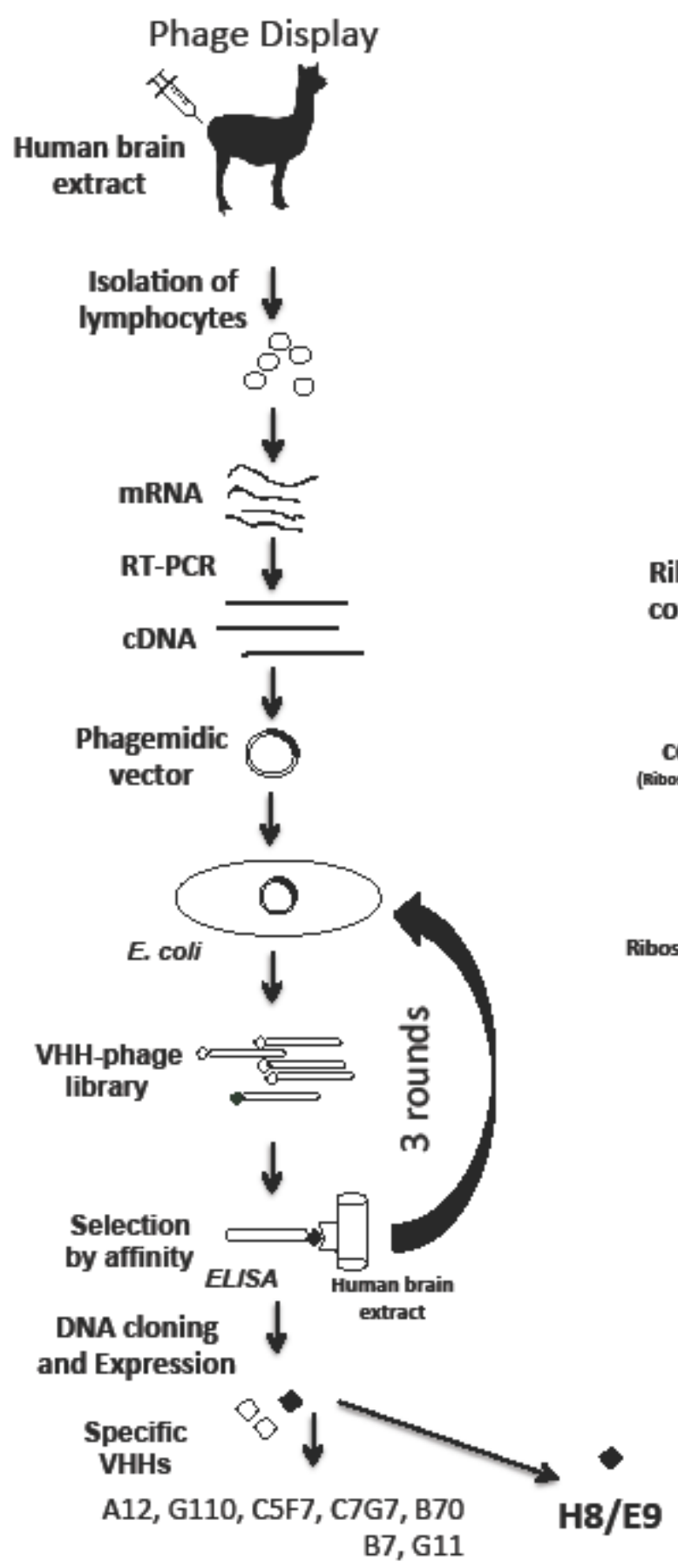

Ribosome Display

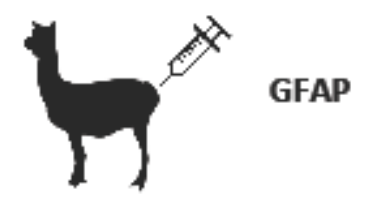

Isolation of

lymphocytes

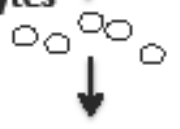

mRNA

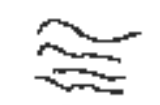

Ribosomal<smiles>C1CC2CCC2C1</smiles>

Ternary

complexes

(Riibosome, mRMA, VHH)

Selection

by affinity

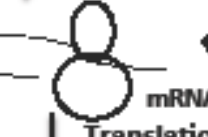

mRNA

Translation

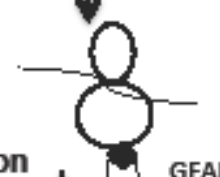

GFAP

몽

Ribosome disruption

Elution

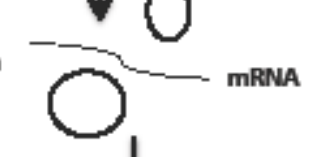
m

Fig. 2. Overview of the selection of the GFAP specific VHHs by phage display and ribosome display. 


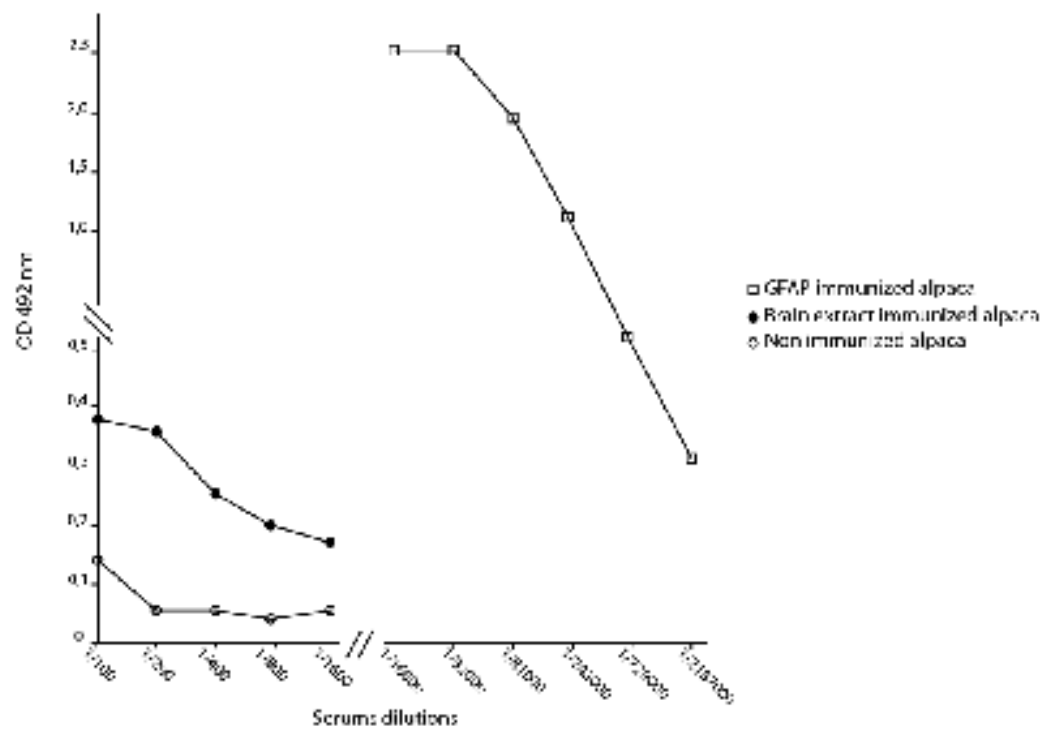

Fig. 3. Polyclonal serum titration on GFAP. 


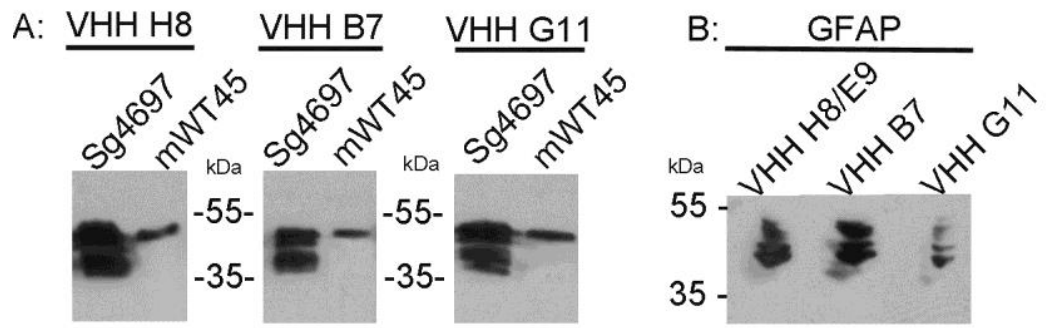

Fig. 4. Western Blot analysis of recombinant VHHs H8/E9, B7 and G11 A:) on human (Sg 4697) and mouse (mWT45) brain extracts, and B:) on purified GFAP. 


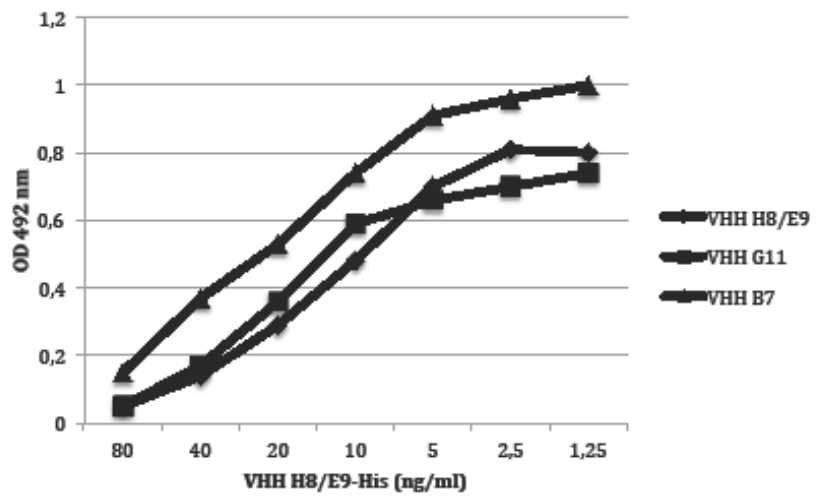

Fig. 5. Competition for binding to GFAP between the different VHHs.

Polystyrene plates were coated with GFAP. His tagged VHH E8/H9 at different concentrations was added, followed by strep-tagged VHH H8/E9, B7, and G11 and revealed by an anti-strep tag $\mathrm{mAb}$. 


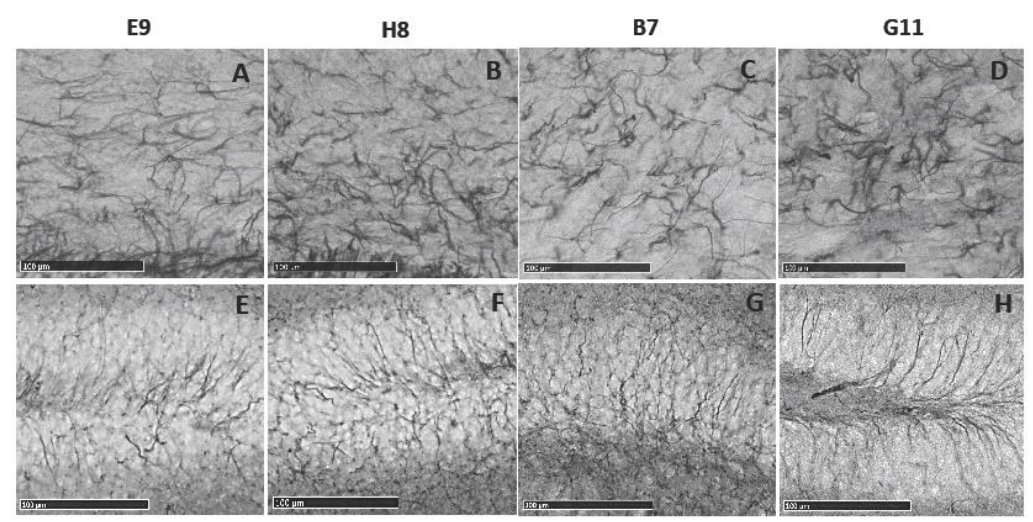

Fig. 6. Immunolabeling of astrocytes by VHHs on mouse brain sections.

Immunostaining with E9 (obtained by ribosome-display) and with H8, B7, G11 (obtained by phage-display). (A, B, C \& D) Protoplasmic astrocytes with short ramifications. (E, F, G \& H) Fibrous astrocytes with thin and long processes. 
Table 1. Affinity of VHHs determined by ELISA using GFAP as target antigen.

\begin{tabular}{|c|c|}
\hline VHHs & $\begin{array}{c}\text { Affinity } \\
(\mathrm{nM})\end{array}$ \\
\hline B7 & 0.71 \\
\hline G11 & 4.6 \\
\hline H8/E9 & 5.6 \\
\hline E3 & $>\mu \mathrm{M}$ \\
\hline A10 & 3.1 \\
\hline
\end{tabular}

